\title{
A Review on Architecture, Issues, Challenges and Applications in Underwater Wireless Sensor Network
}

\author{
Gaurav Rai ${ }^{1}$, Ravi Kumar Malik ${ }^{2}$ \\ ${ }^{1}$ Research Scholar, ${ }^{2}$ Assistant Professor \\ Geeta Engineering College, Panipat, Haryana, India
}

\begin{abstract}
Underwater Wireless Sensor Network (UWSN) is an emerging technique for various underwater applications such as marine climate observation, pollution tracking, disaster prevention, underwater surveillance etc. And, each of these applications require Sensor Nodes (SNs) to effectively provide accurate sensed data. A node must know its own location before sending data to its neighbour. The need for location arises because the number of nodes is very large and it is not possible for the base station to find the nodes' positions, so the individual node is required to send location information along with the observed data to provide exact location to the user, which means the node must localize itself. However, due to the complex environment, it is very challenging to transmit the collected data to the base station on the surface quickly and effectively. An energy efficient routing protocol plays a vital role in data transmission. However, due to the specific characteristics of UWSNs, such as dynamic structure, narrow bandwidth, rapid energy consumption, and high latency, it is difficult to build routing protocols for UWSNs. In this article, we focus on surveying
\end{abstract}

architecture, challenges, opportunities as well as various applications in UWSN.

Keyword: UWSN, sensor nodes, application of UWSN, Acoustic signals.

\section{INTRODUCTION}

SNs are mostly battery-powered. When deployed under the water, SNs can hardly be replaced or recharged in this harsh environment, and ambient energy harvesting combined with super-capacitors is promising to be applied in UWSNs in the future [1]. Therefore, energy efficiency is fundamental when designing routing protocols for gathering sensory data and routing these data to the sink node. It is worth mentioning that most SNs drifts along with water dynamics, which makes that the task of localising SNs is very expensive and error-prone [2-4]. This means that packets routing in an end-to-end manner may not be energy efficient when the network topology may change frequently and dramatically. In this paper, we will study architecture, challenges, opportunities as well as various applications in UWSN.

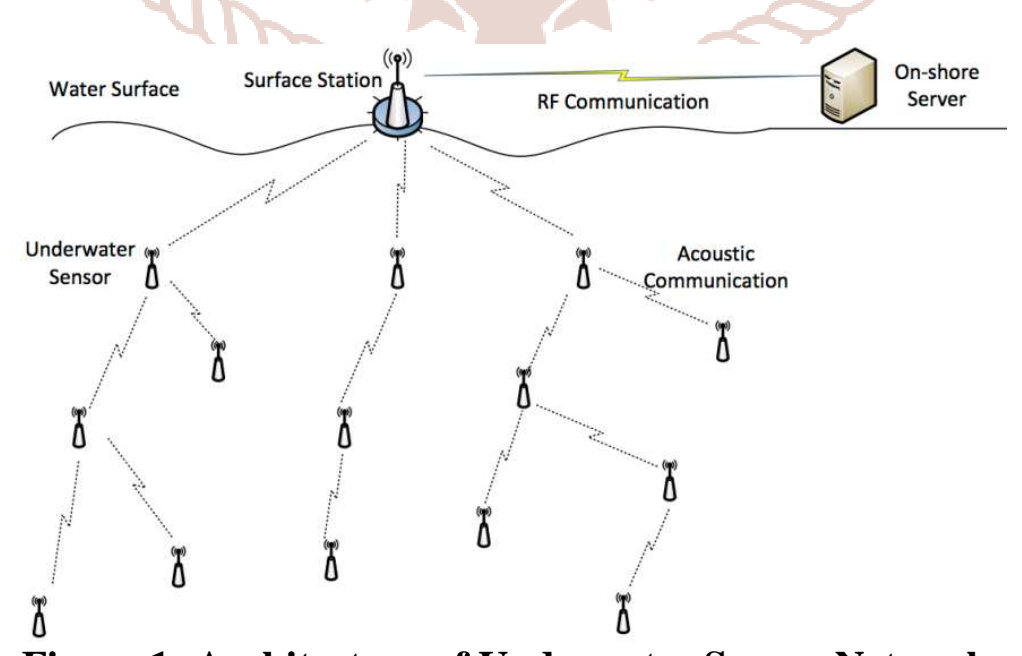

Figure 1: Architecture of Underwater Sensor Network 


\section{Coverage in UWSN}

Coverage refers to monitoring of field in underwater through SNs[5]. Overall coverage provides you better chances to detect any event. Coverage depends on node deployment. There are various kinds. Various factors There are several attributes such as type of network, sensing model, node mobility, types of nodes that affect the achievable coverage in underwater. These are briefly described below.

i. Type of Networks

Generally, two types of network has been used. first, centralized network, in which a central node directly interact with each and every node available in the network. The centralized network consumes more energy. So, overall network lifetime of the system get reduced. Second, distributed network, in which nodes form a network among themselves. These networks consumes less energy as the node can communicate with neighbour nodes for data receiving and data transmission.

ii. Sensing Model

Mostly, two types of sensing models used are boolean model and probabilistic model. In boolean model, after a certain distance node's are not able to sense. In 2-Dimensional underwater scenario, sensing radius takes a disk shape and in 3-Dimensional scenario, it takes a spherical shape. In probabilistic model, the sensing behaviour of nodes is certain it reduces for a little time span.

iii. Node Mobility

In UWSN, node mobility can be of two types. One is a mobile SN and the other is a static $\mathrm{SN}$, but it can move with the help of mobile medium. Nodes equipped with movability are a desired feature.

iv. Types of Nodes

Broadly, types of SNs are used in UWSN are of two types. first, SNs with scalar sensors (like temperature, pressure etc.) have omnidirectionality due to which the sensing locus forms a disk (2D) or a sphere (3D). And, the other kind of nodes are multimedia sensors with a directional camera. These sensors have unidirectionality and has good sensing range towards a particular direction.

v. Energy Consumption

The energy of the node is the central factor that affects all other performance parameters. Due to complete energy draining if a node dies, it results in the formation of coverage holes which makes the system less efficient in offering the coverage.

vi. Deployment Techniques

SNs deployment is a very crucial process as it decides the achievable sensing and communication coverage in UWSN [6], [7]. It is generally of two types. First, 2D coverage in which involves SNs are deployed either on the water surface or on the seabed. But it is prone to manipulation by human intervention. Secondly, a 3D coverage in which the nodes are deployed in underwater 3D spaces.

\section{Underwater Wireless Sensor Network Architecture}

On basis of design, common architectures of UWSN are as follows:

i. One-dimensional Architecture of UWSN

In One-dimensional UWSN the nodes can communicate using acoustic, Radio Frequency (RF), or optical communication. The nature of topology in One-dimensional UWSN is star. It refers to a network where the SNs are deployed autonomously and each sensor node is a stand-alone network itself which is responsible for sensing, processing, and transmitting the information to the remote station [5]. It can be an Autonomous Underwater Vehicle (AUV) which dives inside the water, sense the data and transfer the information to the remote station.

ii. Two-dimensional Architecture of UWSN. Two-dimensional UWSN architecture refers to a network where a group of SNs forms a cluster. Each cluster has a Cluster Head (CH). Each member of the cluster gathers the data and communicates it to the $\mathrm{CH}$. After collecting data, the $\mathrm{CH}$ relays it to the sink. For the cluster of nodes, the network arrangement can be dynamic (star, mesh, or ring) depending on the application requirement [6].

iii. 3D-UWSN Architecture. In this architecture, the sensors are deployed underwater in the form of clusters and are anchored at different depths. Different communication scenarios in this architecture are: (i) intercluster communication of nodes, (ii) intracluster (sensor-anchor node) communication, and (iii) CH-sink communication. Acoustic, optical, and RF links for communication can be used. 
iv. $\quad 4 D-U W S N$ Architecture It is a combination of fixed UWSN and mobile UWSNs. The mobile UWSN consists of remotely operative underwater vehicles (ROVs) to collect data from the anchor nodes and relay the data to the remote station. ROVs can be autonomous submersible robots, vehicles, ships, and even submarines. Each underwater sensor node can be autonomous in relaying the data directly to ROVs depending on how close that particular sensor node is to the ROV. The communication scenario between ROV and underwater sensor node depends on the distance and data between them and either acoustic or radio can be used. As the transmission is to be directly relayed to ROV, the sensors which have large data and are close to ROVs can use radio links while the sensors which have small data to transmit or are far from ROV can use acoustics links [7, 8].

\section{Challenges and Opportunities in UWSN}

UWSN is a promising new field and may help in exploring the unexplored world that lies underwater. Still there are many challenges and opportunities that are as follows:

i. Unpredictable Environment. Underwater conditions are extremely unpredictable. The anonymous high water pressure, unpredictable underwater activities, and uneven depths of the underwater surface make it difficult to design and deploy UWSNs.

ii. Design and Deployment. Due to the unpredictable underwater environment, it is extremely difficult to deploy the network underwater which works reliably and wirelessly. The current tethered technology allows constrained communication but it incurs significant cost of deployment, maintenance, and device recovery to cope with volatile undersea conditions.

iii. Unscalability. Traditional underwater exploration relies on either a single high-cost underwater device or a small-scale underwater network. Neither existing technology is suitable for applications covering a large area. Enabling a scalable underwater sensor network technology is essential for exploring a huge underwater space.

iv. Unreliable Information. Underwater nodes are in continuous motion due to the water currents; thus locating nodes underwater becomes much more crucial. Traditional positioning and localization systems do not work underwater. Therefore, underwater conditions dismantle the location of the nodes and the network topology which ultimately makes the information transmission unreliable.

v. Requirement of Novel Protocols for UWSNs. In underwater communication, the medium of communication is water, unlike air as in terrestrial sensor networks. Therefore, terrestrial sensor network communication protocols get void underwater. Mostly, acoustic signals are used for underwater communication over large distances, while radios are considered for short-distance, water surface communication. But radio signals transmit for long distances at extra low frequencies, which requires large antennas and high transmission power [1], which can decrease the overall network lifetime of UWSNs. Moreover, the propagation delay of acoustic communication is very high compared to RF communication; hence many algorithms and protocols for terrestrial WSN cannot be adapted directly to UWSN.

vi. Low Data Rates. Radio frequency (RF) communications are not effective in underwater communication due to medium effect on communication. Water absorbs much of the RF energy and hence only very short range communication is allowable using $\mathrm{RF}$ [4]. Instead, acoustic communication is being used to transmit pulse signals and low fidelity information underwater due to its low bandwidth. Potential UWSN applications such as measuring the amount of pollution from a fishing farm at the seabed [9] require transmitting lots of data. However, with such low frequencies, it requires a lot of time to send such dynamic data.

vii. Physical Damage to Equipment. The sensors used in underwater devices are susceptible to routine underwater challenges, for example, algae collection on camera lens [10] and salt accumulation, decreasing the effectiveness of sensors and so forth.

viii. Cost. Finally, the energy requirements and cost of UASNs are high compared to higher power and regular battery replenishing techniques are quite costly. The amount of challenges in designing of UWSNs makes it 
an interesting area for researchers to work on. With the advancement in sensor and wireless technologies, UWSNs have attracted a lot of researchers and have contributed significantly to this field. However, the window is still wide open for upcoming research and opportunities.

\section{Factors Affecting Underwater Connectivity}

There are several factors that affect the connectivity in any network. These factors are summarized in Fig. 10. Some of which are vividly explained in this section.

1. Sink Architecture

If there are multiple sinks, then the nodes will have an alternative path to connect to the sink [11]. Further, the probability of loosing connectivity due to failure of any intermediate node in the connection link gets reduced. Thus, the connectivity gets enhanced. In Underwater communication, when the distance of coverage is very large, the multi sink architecture can provide very reliable connectivity.

2. Topology

Proper choice of topology makes the connectivity more reliable. For example, in case star topology, there is only single hop communication, in case of mesh topology, multi-hop is possible and clustering topology is a combination of multimesh networks [9].

3. Signal Propagation Loss

The loss of connectivity is proportional to the loss of signal. The signal loss is due to signal traversing a significant distance, or due to absorption of particles or molecules in the medium, or due to weather conditions.

4. Reachability

It is a sensitive metric that quantifies the ability of the network to communicate (especially in sparse networks) [8], [9].It is the fraction of node pairs in the total number of nodes. Less reachability refers to a situation where the number of node pairs to form a link are less; consequently the connectivity is poor.

5. Shadow Zones

These are the spatial locations where communication signal is practically void. These zones are quite common, especially when the area of coverage is very large and it can be expected in areas where there is a high signal attenuation [10], [11]. Underwater is the medium where shadow zones can be expected because of having a wide area to cover.

6. Internodal Distance

In UASN, nodes after initial deployment are lowered to a calculated depths. However, in doing so, increasing the distance between nodes reduces connectivity, whereas decreasing the node distance enhances the coverage overlap. Thus, there is a trade off between coverage and connectivity. However, a balance of both can be achieved by keeping communication radius more than sensing radius.

\section{Underwater Wireless Sensor Network Application Classification}

UWSN applications are rapidly gaining popularity for enabling advances in the area of ocean monitoring and observatory systems, deep sea surveillance, tracking of various entities of the aquatic environment, and unearthing resources [12]. UWSNs find their application in fields like offshore oil and gas extraction, oil spills, military surveillance and reconnaissance, mine detection, pollution monitoring, natural calamities like tsunami and hurricane forecast, coral reef and habitat monitoring of marine life, and fish farming. A comprehensive classification of potential UWSN applications is shown in Figure 2.

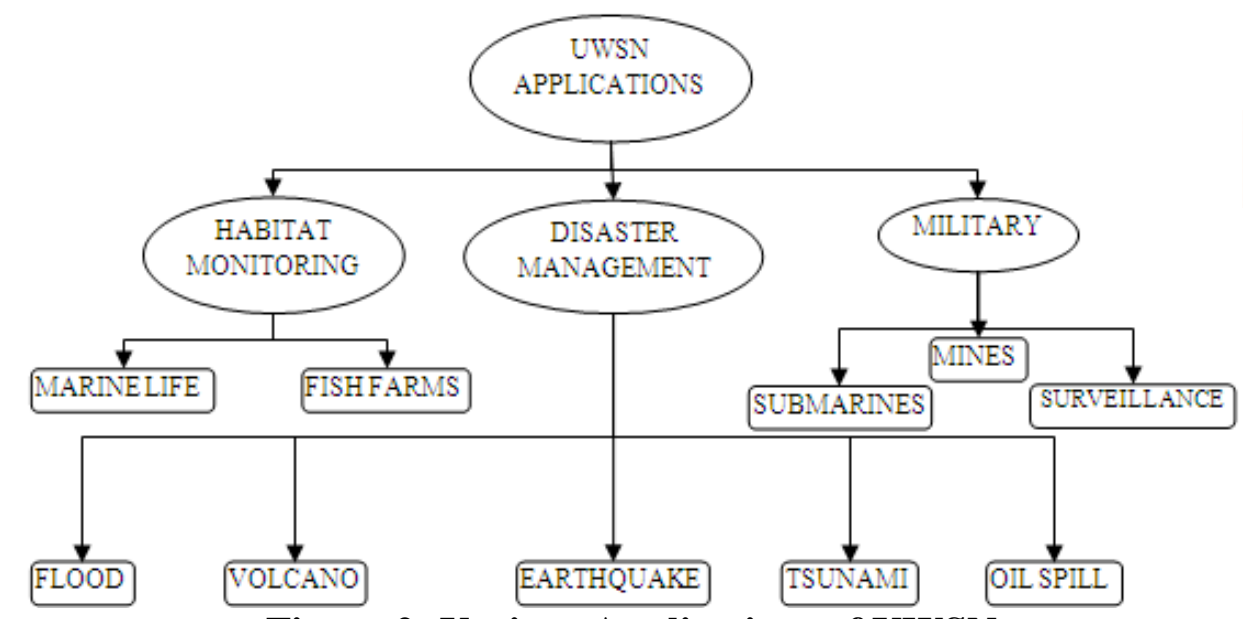

Figure 2: Various Applications of UWSN 


\section{CONCLUSION}

When deployed under the water, SNs can hardly be replaced or recharged in this harsh environment, and ambient energy harvesting combined with supercapacitors is promising to be applied in UWSNs. Therefore, energy efficiency is fundamental when designing routing protocols for gathering sensory data and routing these data to the sink node. However, due to the complex environment, it is very challenging to transmit the collected data to the base station on the surface quickly and effectively. An energy efficient routing protocol plays a vital role in data transmission. However, due to the specific characteristics of UWSNs, such as dynamic structure, narrow bandwidth, rapid energy consumption, and high latency, it is difficult to build routing protocols for UWSNs. In future, we will propose a routing protocol which will enhance the lifetime of the network.

\section{REFERENCES}

1. X. Feng, Z. Wang, G. Han, W. Qu and A. Chen, "Distributed Receiver-Oriented Adaptive Multichannel MAC for Underwater Sensor Networks," in IEEE Access, vol. 6, pp. 1166611675, 2018.

2. P. Gjanci, C. Petrioli, S. Basagni, C. A. Phillips, L. Bölöni and D. Turgut, "Path Finding for Maximum Value of Information in Multi-Modal Underwater Wireless Sensor Networks," in IEEE Transactions on Mobile Computing, vol. 17, no. 2, pp. 404-418, 1 Feb. 2018.

3. H. Yang, Y. Zhou, Y. Hu, B. Wang and S. Kung, "Cross-Layer Design for Network Lifetime Maximization in Underwater Wireless Sensor Networks," 2018 IEEE International Conference on Communications (ICC), Kansas City, MO, USA, 2018, pp. 1-6.

4. M. A. Yaqub, M. T. R. Khan, S. H. Ahmed and D. Kim, "Receiver-initiated dynamic duty cycle scheduling schemes for underwater wireless sensor networks," 2018 15th IEEE Annual Consumer Communications \& Networking
Conference (CCNC), Las Vegas, NV, 2018, pp. 16.

5. R. Martin and S. Rajasekaran, "Data centric approach to analyzing security threats in Underwater Sensor Networks," OCEANS 2016 MTS/IEEE Monterey, Monterey, CA, 2016, pp. 16.

6. D. Das and P. M. Ameer, "Energy efficient geographic clustered multi-hop routing for underwater sensor networks," TENCON 2017 2017 IEEE Region 10 Conference, Penang, 2017, pp. 409-414.

7. M. Aslam et al., "Energy Efficient Cubical layered Path Planning Algorithm (EECPPA) for acoustic UWSNs," 2017 IEEE Pacific Rim Conference on Communications, Computers and Signal Processing (PACRIM), Victoria, BC, 2017, pp. 16.

8. B. Zhang, Y. Wang, H. Wang, X. Guan and Z. Zhuang, "Tracking a Duty-Cycled Autonomous Underwater Vehicle by Underwater Wireless Sensor Networks," in IEEE Access, vol. 5, pp. 18016-18032, 2017.

9. N. Javaid, S. Cheema, M. Akbar, N. Alrajeh, M. S. Alabed and N. Guizani, "Balanced Energy Consumption Based Adaptive Routing for IoT Enabling Underwater WSNs," in IEEE Access, vol. 5, pp. 10040-10051, 2017.

10. J. Kartha, A. Jabbar, A. Baburaj and L. Jacob, "Maximum lifetime routing in underwater sensor networks using mobile sink for delay-tolerant applications," TENCON 2015 - 2015 IEEE Region 10 Conference, Macao, 2015, pp. 1-6.

11. G. Han, J. Jiang, N. Bao, L. Wan and M. Guizani, "Routing protocols for underwater wireless sensor networks," in IEEE Communications Magazine, vol. 53, no. 11, pp. 72-78, November 2015.

12. Z. Zhou, B. Yao, R. Xing, L. Shu and S. Bu, "ECARP: An Energy Efficient Routing Protocol for UWSNs in the Internet of Underwater Things," in IEEE Sensors Journal, vol. 16, no. 11, pp. 40724082, June1, 2016. 